\title{
Modeling Cell-to-Cell Spread of HIV-1 with Nonlocal Infections
}

\author{
Xiaoting Fan $\mathbb{D}^{1}{ }^{1}$ Yi Song $\mathbb{D}^{2,3}$ and Wencai Zhao $\mathbb{D}^{2,3}$ \\ ${ }^{1}$ College of Applied Sciences, Beijing University of Technology, Beijing 100124, China \\ ${ }^{2}$ College of Mathematics and Systems Science, Shandong University of Science and Technology, Qingdao 266590, China \\ ${ }^{3}$ State Key Laboratory of Mining Disaster Prevention and Control Co-Founded by Shandong Province and the Ministry of Science \\ and Technology, Shandong University of Science and Technology, Qingdao 266590, China
}

Correspondence should be addressed to Yi Song; songyi_sy@sina.com

Received 18 November 2017; Accepted 10 July 2018; Published 1 August 2018

Academic Editor: Dimitri Volchenkov

Copyright (c) 2018 Xiaoting Fan et al. This is an open access article distributed under the Creative Commons Attribution License, which permits unrestricted use, distribution, and reproduction in any medium, provided the original work is properly cited.

\begin{abstract}
This paper is devoted to develop a nonlocal and time-delayed reaction-diffusion model for HIV infection within host cell-to-cell viral transmissions. In a bounded spatial domain, we study threshold dynamics in terms of basic reproduction number $\mathscr{R}_{0}$ for the heterogeneous model. Our results show that if $\mathscr{R}_{0}<1$, the infection-free steady state is globally attractive, implying infection becomes extinct, while if $\mathscr{R}_{0}>1$, virus will persist in the host environment.
\end{abstract}

\section{Introduction and Model Derivation}

Infectious diseases have been threatening human health; infectious diseases such as smallpox, cholera, and acquired immune deficiency syndrome (AIDS) have brought great disaster to the national economy of a country and people's livelihood. In order to control the spread of infectious diseases, researchers have proposed a great deal of mathematical models to study the evolution behavior of infectious diseases from different views [1-7]. Mathematical models have been confirmed to be an effective and valuable approach to understand the dynamical behavior of infectious diseases [8-11]. Many diseases are caused by a virus, for example, human immunodeficiency virus (HIV) can destroy the immune cells, reduce human immunity, and ultimately lead to AIDS. A lot of mathematical models have been built to explain the virus infection process from different views [12-15]. In recent years, the virus infection dynamical models incorporating spatial dispersion $[16,17]$ have received widely attentions. For example, under spatial domain equipped with suitable boundary conditions, $\mathrm{K}$. Wang and W. Wang [18] established the existence of travelling wave solutions via the geometric singular perturbation method and the spatial domain is assumed to be one dimensional, that is, $(x, t) \in(-\infty, \infty) \times(0,+\infty)$. And then, Brauner et al. [19] extended the works [18] to a two- dimensional square domain $(0, l) \times(0, l)$ with periodic boundary conditions by introducing a new parameter $\lambda_{0}$ which is the largest eigenvalue of some Sturm-Liouville problem and taking the heterogeneous reproductive ratio into account. Recently, Wang et al. [20] noticed that a realistic domain should be bounded but is typically not a square, under no-flux boundary condition (homogenous Neumann boundary condition) in a general bounded domain $\Omega \subset R^{n}$ with smooth boundary $\partial \Omega$, and threshold dynamics of the model were investigated by appealing to the theory of uniform persistence and the comparison theorem. Some further developments have been performed on the virus infection dynamical models with diffusion term (see, for example, Hattaf and Yousfi [21], Wang and Ma [22-24], Lai and Zou [25], Wang and Xu [26], and Wang et al. [27]).

It is widely known that virus-to-cell transmission and direct cell-to-cell transmission are two predominant infection modes in within-host environment (see, for example, $[28,29])$. The virus-to-cell transmission has iterative process: the binding of virus to a receptor on the surface of $\mathrm{CD} 4^{+} \mathrm{T}$ cells, the fusion of virus with host cells and then the release of genetic materials, the transcription of genetic materials in an infected cell, the assembling of virus inside the infected cells, and budding through the membrane of the infected cells [30]. The cell-to-cell transmission process is due to that a large number of viruses can be transferred 
from infected cells to uninfected cells through the formation of virally induced structures termed virological synapses [31]. In order to examine the effects of both diffusion and spatial heterogeneity, Wang et al. [32] proposed the following virus infection dynamical model incorporating cell-to-cell transmission under the homogeneous Neumann boundary condition:

$$
\begin{aligned}
\frac{\partial U(x, t)}{\partial t}= & \xi(x)-d_{U}(x) U(x, t)-\beta_{1}(x) U(x, t) \omega(x, t) \\
& -\beta_{2}(x) U(x, t) V(x, t), \\
\frac{\partial V(x, t)}{\partial t}= & \beta_{1}(x) U(x, t) \omega(x, t)+\beta_{2}(x) U(x, t) V(x, t) \\
& -d_{V}(x) V(x, t), \\
\frac{\partial \omega(x, t)}{\partial t}= & D_{\omega} \Delta \omega+k(x) V(x, t)-d_{\omega}(x) \omega(x, t) .
\end{aligned}
$$

In model (1), $\xi(x)$ is the production rate of newly produced uninfected cells. $d_{U}(x), d_{V}(x)$, and $d_{U}(x)$ represent the death rates of uninfected cells, infected cells, and free viruses, respectively. $\beta_{1}(x)$ denotes the transmission coefficient for virus-to-cell infection. $\beta_{2}(x)$ represents the transmission coefficient for cell-to-cell infection. $k(x)$ denotes the production rate of virus due to the lysis of infected cells. $D_{\omega}$ is the diffusion coefficient and $\Delta$ is Laplacian. For model (1), the virus dynamics are fully determined by an important parameter which is called basic reproduction number $\mathscr{R}_{0}$, in the following sense: if $\mathscr{R}_{0}<1$, the infection-free steady state is globally asymptotically stable, while if $\mathscr{R}_{0}>1$, the model is uniformly persistent and the infection steady state is globally asymptotically stable.

The mobility of cells in the latent period will result in a delay term with spatial averaging on the spatial habitat $\Omega$ [33]. To formulate this process with the latency properly, we introduce an infection age variable $a_{1}$. The infected cells can be divided into two epidemiological categories: latently infected cells $(I=I(x, t))$ and actively infected cells $(V=$ $V(x, t))$. Let $M\left(t, a_{1}, x\right)$ represent the density of cells with infection age $a_{1}$ at time $t$ and habitat $x$. From the biological perspective, we assume that all populations remain confined to the region $\Omega$ for all time and subject to noflux boundary condition for $M\left(t, a_{1}, x\right)$ :

$$
\left[D\left(a_{1}, x\right) \nabla M\left(t, a_{1}, x\right)\right] \cdot v=0,
$$

where $v$ is the outward normal to the smooth boundary $\partial \Omega$. We adopt the standard model on describing age-structured population with spatial diffusion [34]. Then

$$
\begin{aligned}
\frac{\partial M\left(t, a_{1}, x\right)}{\partial t}+\frac{\partial M\left(t, a_{1}, x\right)}{\partial a_{1}}=\nabla & \cdot\left[D\left(a_{1}, x\right) \nabla M\left(t, a_{1}, x\right)\right] \\
& -d_{V}(x) M\left(t, a_{1}, x\right)
\end{aligned}
$$

In (3), $\nabla M\left(t, a_{1}, x\right)$ is the gradient of $M\left(t, a_{1}, x\right)$ with respect to the spatial variable $x$ and $\nabla \cdot\left[D\left(a_{1}, x\right) \nabla M\left(t, a_{1}, x\right)\right]$ represents the divergence of $D\left(a_{1}, x\right) \nabla M\left(t, a_{1}, x\right) . D\left(a_{1}, x\right)$ represents the diffusion rate of cells at age $a_{1}$ and habitat $x$. $d_{V}(x)$ is the natural death rate which is independent of the infection age $a_{1}$. Let $\tau$ be the average incubation period. Then

$$
I(x, t)=\int_{0}^{\tau} M\left(t, a_{1}, x\right) d a_{1}, \quad(x, t)=\int_{\tau}^{\infty} M\left(t, a_{1}, x\right) d a_{1} .
$$

In order to make the model mathematically tractable yet without losing the main characteristics, we make some assumptions for function $D\left(a_{1}, x\right)$ as follows:

$$
D\left(a_{1}, x\right)= \begin{cases}D_{I}(x), & \text { for } t \geq 0, a_{1} \in[0, \tau] \text { and } x \in \Omega, \\ D_{V}(x), & \text { for } t \geq 0, a_{1} \in[\tau, \infty] \text { and } x \in \Omega .\end{cases}
$$

Integrating both sides of (3) from 0 to $\tau$ and from $\tau$ to $\infty$, it then follows that

$$
\begin{aligned}
\frac{\partial I(x, t)}{\partial t}= & \nabla \cdot\left[D_{I}(x) \nabla I(x, t)\right]-d_{V}(x) I(x, t) \\
& -M(t, \tau, x)+M(t, 0, x),
\end{aligned}
$$

and

$$
\begin{aligned}
\frac{\partial V(x, t)}{\partial t}= & \nabla \cdot\left[D_{V}(x) \nabla V(x, t)\right]-d_{V}(x) V(x, t) \\
& +M(t, \tau, x)-M(t, \infty, x) .
\end{aligned}
$$

For biological reasons, we assume that $M(t, \infty, x)=0$ (see, for example, [35]), since the recruitment of newly infected cells $(M(t, 0, x))$ is divided into two parts: the contact of uninfected cells and virus and the contact of uninfected cells and infected cells. Thus, adopting BeddingtonDeAngelis functional response which was firstly proposed by $[36,37]$ leads to the following condition:

$$
\begin{aligned}
M(t, 0, x)= & \frac{\beta_{1}(x) U(x, t) \omega(x, t)}{1+a_{1}(x) \omega(x, t)+b_{1}(x) U(x, t)} \\
& +\frac{\beta_{2}(x) U(x, t) V(x, t)}{1+a_{2}(x) V(x, t)+b_{2}(x) U(x, t)},
\end{aligned}
$$

where $a_{1}(x)$ and $a_{2}(x)$ are the measure of virus interference during infection and $b_{1}(x)$ and $b_{2}(x)$ determine how fast the infection rate approaches its saturation value (see, for example, $[36,37])$.

In the following, we determine $M(t, \tau, x)$ by the method of characteristics. Let $v\left(r, a_{1}, x\right)=M\left(a_{1}+r, a_{1}, x\right)$ with $r \geq 0$. For $a_{1} \in[0, \tau]$, it then follows that 


$$
\begin{aligned}
\frac{\partial v\left(r, a_{1}, x\right)}{\partial a_{1}}= & {\left[\frac{\partial M\left(r, a_{1}, x\right)}{\partial t}+\frac{\partial M\left(r, a_{1}, x\right)}{\partial a_{1}}\right]_{t=a_{1}+r} } \\
= & \nabla \cdot\left[D_{I}(x) \nabla M\left(a_{1}+r, a_{1}, x\right)\right] \\
& -d_{V}(x) M\left(a_{1}+r, a_{1}, x\right) \\
= & \nabla \cdot\left[D_{I}(x) \nabla v\left(r, a_{1}, x\right)\right] \\
& -d_{V}(x) v\left(r, a_{1}, x\right), \\
v(r, 0, x)= & \frac{\beta_{1}(x) U(r, x) \omega(r, x)}{1+a_{1}(x) \omega(r, x)+b_{1}(x) U(r, x)} \\
& +\frac{\beta_{2}(x) U(r, x) V(r, x)}{1+a_{2}(x) V(r, x)+b_{2}(x) U(r, x)} .
\end{aligned}
$$

Regarding $r$ as a parameter and solving the above equation, we obtain that

$$
\begin{aligned}
v\left(r, a_{1}, x\right)= & \int_{\Omega} \frac{\Gamma\left(a_{1}, x, y\right) \beta_{1}(y) U(y, r) \omega(y, r)}{1+a_{1}(y) \omega(y, r)+b_{1}(y) U(y, r)} d y \\
& +\int_{\Omega} \frac{\Gamma\left(a_{1}, x, y\right) \beta_{2}(y) U(y, r) \omega(y, r)}{1+a_{2}(y) \omega(y, r)+b_{2}(y) U(y, r)} d y
\end{aligned}
$$

where $\Gamma\left(a_{1}, x, y\right)$ is the fundamental solution associated with the partial differential operator $\nabla \cdot\left[D_{I}(\cdot) \nabla\right]-d_{V}(\cdot)$, which satisfies $\int_{\Omega} \Gamma\left(a_{1}, x, y\right) d y=1, \forall x \in \Omega . \Gamma\left(a_{1}, x, y\right)=\Gamma$ $\left(a_{1}, y, x\right)$, and $\Gamma\left(a_{1}, x, y\right)>0 \quad \forall x \in \Omega, \quad x \neq y \quad$ (see, [38], Chapter 1).

Since $M(t, \tau, x)=v(t-\tau, \tau, x), \forall t \geq \tau$, we get the formula for $M(t, \tau, x)$ for $a_{1}=\tau$ :

$$
\begin{aligned}
M(t, \tau, x)= & \int_{\Omega} \Gamma(\tau, x, y) \frac{\beta_{1}(y) U(y, t-\tau) \omega(y, t-\tau)}{1+a_{1}(y) \omega(y, t-\tau)+b_{1}(y) U(y, t-\tau)} d y \\
& +\int_{\Omega} \Gamma(\tau, x, y) \frac{\beta_{2}(y) U(y, t-\tau) V(y, t-\tau)}{1+a_{2}(y) V(y, t-\tau)+b_{2}(y) U(y, t-\tau)} d y .
\end{aligned}
$$

It then follows that

$$
\begin{aligned}
\frac{\partial I(x, t)}{\partial t}= & \nabla \cdot\left[D_{I}(x) \nabla I(x, t)\right]-d_{V}(x) I(x, t) \\
& -\int_{\Omega} \frac{\Gamma(\tau, x, y) \beta_{1}(y) U(y, t-\tau) \omega(y, t-\tau)}{1+a_{1}(y) \omega(y, t-\tau)+b_{1}(y) U(y, t-\tau)} d y \\
& -\int_{\Omega} \frac{\Gamma(\tau, x, y) \beta_{2}(y) U(y, t-\tau) V(y, t-\tau)}{1+a_{2}(y) V(y, t-\tau)+b_{2}(y) U(y, t-\tau)} d y \\
& +\frac{\beta_{1}(x) U(x, t) \omega(x, t)}{1+a_{1}(x) \omega(x, t)+b_{1}(x) U(x, t)} \\
& +\frac{\beta_{2}(x) U(x, t) V(x, t)}{1+a_{2}(x) V(x, t)+b_{2}(x) U(x, t)}
\end{aligned}
$$

$$
\begin{aligned}
\frac{\partial V(x, t)}{\partial t}= & \nabla \cdot\left[D_{V}(x) \nabla V(x, t)\right]-d_{V}(x) V(x, t) \\
& +\int_{\Omega} \frac{\Gamma(\tau, x, y) \beta_{1}(y) U(y, t-\tau) \omega(y, t-\tau)}{1+a_{1}(y) \omega(y, t-\tau)+b_{1}(y) U(y, t-\tau)} d y \\
& +\int_{\Omega} \frac{\Gamma(\tau, x, y) \beta_{2}(y) U(y, t-\tau) V(y, t-\tau)}{1+a_{2}(y) V(y, t-\tau)+b(y)_{2} U(y, t-\tau)} d y .
\end{aligned}
$$

With all these assumptions, the HIV infection dynamics modeling cell-to-cell with nonlocal infections can be described by the following nonlocal and time-delayed reaction-diffusion equations:

$$
\begin{aligned}
\frac{\partial U(x, t)}{\partial t}= & \nabla\left[D_{U}(x) \nabla U(x, t)\right]+\xi(x)-d_{U}(x) U(x, t) \\
& -\frac{\beta_{1}(x) U(x, t) \omega(x, t)}{1+a_{1}(x) \omega(x, t)+b_{1}(x) U(x, t)} \\
& -\frac{\beta_{2}(x) U(x, t) V(x, t)}{1+a_{2}(x) V(x, t)+b_{2}(x) U(x, t)} \\
\frac{\partial I(x, t)}{\partial t}=\nabla & \cdot\left[D_{I}(x) \nabla I(x, t)\right]-d_{V}(x) I(x, t) \\
& -\int_{\Omega} \frac{\Gamma(\tau, x, y) \beta_{1}(y) U(y, t-\tau) \omega(y, t-\tau)}{1+a_{1}(y) \omega(y, t-\tau)+b_{1}(y) U(y, t-\tau)} d y \\
& -\int_{\Omega} \frac{\Gamma(\tau, x, y) \beta_{2}(y) U(y, t-\tau) V(y, t-\tau)}{1+a_{2}(y) V(y, t-\tau)+b_{2}(y) U(y, t-\tau)} d y \\
& +\frac{\beta_{1}(x) U(x, t) \omega(x, t)}{1+a_{1}(x) \omega(x, t)+b_{1}(x) U(x, t)} \\
& +\frac{\beta_{2}(x) U(x, t) V(x, t)}{1+a_{2}(x) V(x, t)+b_{2}(x) U(x, t)}, \\
\frac{\partial V(x, t)}{\partial t}=\nabla & \cdot\left[D_{V}(x) \nabla V(x, t)\right]-d_{V}(x) V(x, t) \\
& +\int_{\Omega} \frac{\Gamma(\tau, x, y) \beta_{1}(y) U(y, t-\tau) \omega(y, t-\tau)}{1+a_{1}(y) \omega(y, t-\tau)+b_{1}(y) U(y, t-\tau)} d y \\
& +\int_{\Omega} \frac{\Gamma(\tau, x, y) \beta_{2}(y) U(y, t-\tau) V(y, t-\tau)}{1+a_{2}(y) V(y, t-\tau)+b_{2}(y) U(y, t-\tau)} d y, \\
\frac{\partial \omega(x, t)}{\partial t}= & \cdot\left[D_{\omega}(x) \nabla \omega(x, t)\right]+k(x) V(x, t)-d_{\omega}(x) \omega(x, t) .
\end{aligned}
$$

Since $I(x, t)$ is decoupled from other equations for model (14), we consider the following dynamical model:

$$
\begin{aligned}
\frac{\partial U(x, t)}{\partial t}= & \nabla\left[D_{U}(x) \nabla U(x, t)\right]+\xi(x)-d_{U}(x) U(x, t) \\
& -\frac{\beta_{1}(x) U(x, t) \omega(x, t)}{1+a_{1}(x) \omega(x, t)+b_{1}(x) U(x, t)} \\
& -\frac{\beta_{2}(x) U(x, t) V(x, t)}{1+a_{2}(x) V(x, t)+b_{2}(x) U(x, t)}, \\
\frac{\partial V(x, t)}{\partial t}= & \cdot\left[D_{V}(x) \nabla V(x, t)\right]-d_{V}(x) V(x, t) \\
& +\int_{\Omega} \frac{\Gamma(\tau, x, y) \beta_{1}(y) U(y, t-\tau) \omega(y, t-\tau)}{1+a_{1}(y) \omega(y, t-\tau)+b_{1}(y) U(y, t-\tau)} d y \\
& +\int_{\Omega} \frac{\Gamma(\tau, x, y) \beta_{2}(y) U(y, t-\tau) V(y, t-\tau)}{1+a_{2}(y) V(y, t-\tau)+b_{2}(y) U(y, t-\tau)} d y, \\
\frac{\partial \omega(x, t)}{\partial t}= & \nabla \cdot\left[D_{\omega}(x) \nabla \omega(x, t)\right]+k(x) V(x, t)-d_{\omega}(x) \omega(x, t),
\end{aligned}
$$

and 
for $x \in \Omega, t>0$. We consider a closed environment in the sense that the fluxes for each of these subpopulations are aero, and hence we propose no-flux condition on the boundary:

$$
\begin{aligned}
{\left[D_{U}(x) \nabla U(x, t)\right] \cdot v } & =\left[D_{V}(x) \nabla V(x, t)\right] \cdot v \\
& =\left[D_{\omega}(x) \nabla \omega(x, t)\right] \cdot v \\
& =0, \quad x \in \partial \Omega, t>0,
\end{aligned}
$$

and initial conditions

$$
\begin{array}{r}
U(x, \theta)=\phi_{1}(x, \theta) \geq 0, \\
V(x, \theta)=\phi_{2}(x, \theta) \geq 0, \\
\omega(x, \theta)=\phi_{3}(x, \theta) \geq 0, \\
\theta \in[-\tau, 0] .
\end{array}
$$

We now briefly outline the plan of this paper. In Section 2 , we establish basic reproduction number $\mathscr{R}_{0}$. In Section 3, we study threshold dynamics in terms of basic reproduction number for the heterogeneous model. At last, we give discussions and biological implications in Section 4.

\section{Basic Reproduction Number}

Let $\mathbb{X}:=C\left(\bar{\Omega}, \mathbb{R}^{3}\right)$ be the Banach space with the supremum norm $\|\cdot\|_{\mathbb{X}}$ by $\|\phi\|_{\mathbb{X}}=\sup _{x \in \bar{\Omega}}|\phi(x)|=\sup _{x \in \bar{\Omega}}$ $\sqrt{\phi_{1}^{2}(x)+\phi_{2}^{2}(x)+\phi_{3}^{2}(x)}, \quad \phi=\left(\phi_{1}, \phi_{2}, \phi_{3}\right)^{\mathrm{T}}, \quad$ where $|\cdot|$ denotes the Euclidean norm in $\mathbb{R}^{3}$ and $\mathrm{T}$ denotes the transpose of the vector, and $\mathbb{X}_{+}:=C\left(\bar{\Omega}, \mathbb{R}_{+}^{3}\right)$. We easily get that $\mathbb{X}_{+}$is a closed cone of $\mathbb{X}$.

Define $\mathbb{C}=C([-\tau, 0], \mathbb{X})$ with the norm $\|\phi\|=\sup _{\theta \in[-\tau, 0]}$ $\|\phi(\theta)\|_{\mathbb{X}}$ and $\mathbb{C}_{+}=C\left([-\tau, 0], \mathbb{X}_{+}\right)$, then $\left(\mathbb{C}, \mathbb{C}_{+}\right)$is an ordered Banach space. For any given continuous function $u:[-\tau, \sigma) \rightarrow \mathbb{X}$ for $\sigma>0$, define $u_{t} \in \mathbb{C}_{+}$by $u_{t}(\theta)=u(t+\theta)$, $\theta \in[-\tau, 0]$. Let $\mathbb{Z}:=\mathbb{C}(\bar{\Omega}, \mathbb{R})$ and $\mathbb{Z}_{+}:=\mathbb{C}\left(\bar{\Omega}, \mathbb{R}_{+}\right)$. Suppose that $\mathscr{M}_{1}(t), \mathscr{M}_{2}(t)$, and $\mathscr{M}_{3}(t)$ are the strongly continuous semigroups associated with $\nabla \cdot\left[D_{U}(\cdot) \nabla\right]-d_{U}(\cdot), \nabla$. $\left[D_{V}(\cdot) \nabla\right]-d_{V}(\cdot)$, and $\nabla \cdot\left[D_{\omega}(\cdot) \nabla\right]-d_{\omega}(\cdot)$ subject to homogeneous Neumann boundary conditions, respectively, that is,

$$
\left[\mathscr{M}_{i}(t) \phi\right](x)=\int_{\Omega} \Gamma_{i}(x, y, t) \phi(y) d y
$$

for any $\phi \in \mathbb{Z}, t \geq 0$, and $\mathscr{M}_{i}(0) \phi=\phi, i=1,2,3 . \Gamma_{1}, \Gamma_{2}$, and $\Gamma_{3}$ are the Green functions associated with $\nabla \cdot\left[D_{U}(\cdot) \nabla\right]-d_{U}(\cdot)$, $\nabla \cdot\left[D_{V}(\cdot) \nabla\right]-d_{V}(\cdot)$, and $\nabla \cdot\left[D_{\omega}(\cdot) \nabla\right]-d_{\omega}(\cdot)$, respectively, subject to homogenous Neumann boundary conditions. According to Section 7.1 and Corollary 7.2.3 in [39], it follows that $\mathscr{M}_{i}(t): \mathbb{Z} \rightarrow \mathbb{Z}, i=1,2,3$ is strongly positive and compact for each $t>0$. We can also obtain that $K(t)=$ $\left(\mathscr{M}_{1}(t), \mathscr{M}_{2}(t), \mathscr{M}_{3}(t)\right): \mathbb{X} \rightarrow \mathbb{X}, t \geq 0$, is a strongly continuous semigroup. Let $\mathbb{A}_{i}: D\left(\mathbb{A}_{i}\right) \rightarrow \mathbb{Z}$ be the generator of $\mathscr{M}_{1}$ $(t), \mathscr{M}_{2}(t), \mathscr{M}_{3}(t)$, respectively $(i=1,2,3)$. Then, we get that
$K(t): \mathbb{X} \rightarrow \mathbb{X}$ is a semigroup generated by the operator $\mathbb{A}=$ $\left(\mathbb{A}_{1}, \mathbb{A}_{2}, \mathbb{A}_{3}\right)$ defined on $D(\mathbb{A})=D\left(\mathbb{A}_{1}\right) \times D\left(\mathbb{A}_{2}\right) \times D\left(\mathbb{A}_{3}\right)$.

In view of Lemma 2.2 in [35], model (15) exists a unique infection-free steady state $E_{0}=(\widehat{U}(x), 0,0)$. Linearizing model (15) at the infection-free steady state $E_{0}$, we get the following linearized system:

$$
\begin{aligned}
\frac{\partial v_{1}}{\partial t}= & \nabla \cdot\left[D_{U}(x) \nabla v_{1}(x, t)\right]-d_{U}(x) v_{1}(x, t) \\
& -\frac{\beta_{1}(x) \widehat{U}(x)}{1+b_{1}(x) \widehat{U}(x)} v_{3}(x, t)-\frac{\beta_{2}(x) \widehat{U}(x)}{1+b_{2}(x) \widehat{U}(x)} v_{2}(x, t), \\
\frac{\partial v_{2}}{\partial t}=\nabla \cdot\left[D_{V}(x) \nabla v_{2}(x, t)\right] & \\
& +\int_{\Omega} \Gamma(\tau, x, y) \frac{\beta_{1}(y) \widehat{U}(y)}{1+b_{1}(y) \widehat{U}(y)} v_{3}(t-\tau, y) d y \\
& +\int_{\Omega} \Gamma(\tau, x, y) \frac{\beta_{2}(y) \widehat{U}(y)}{1+b_{2}(y) \widehat{U}(y)} v_{2}(t-\tau, y) d y \\
& -d_{V}(x) v_{2}(x, \mathrm{t}), \\
\frac{\partial v_{3}}{\partial t}= & \nabla \cdot\left[D_{\omega}(x) \nabla v_{3}(x, t)\right]+k(x) v_{2}(x, t)-d_{\omega}(x) v_{3}(x, t),
\end{aligned}
$$

satisfying the following boundary conditions

$$
\begin{aligned}
{\left[D_{U}(x) \nabla v_{1}(x, t)\right] \cdot v } & =\left[D_{V}(x) \nabla v_{2}(x, t)\right] \cdot v \\
& =\left[D_{\omega}(x) \nabla v_{3}(x, t)\right] \cdot v=0, \quad \forall x \in \partial \Omega, t>0 .
\end{aligned}
$$

We consider the following model:

$$
\begin{aligned}
\frac{\partial v_{1}}{\partial t}= & \nabla \cdot\left[D_{U}(x) \nabla v_{1}(x, t)\right]-d_{U}(x) v_{1}(x, t) \\
& -\frac{\beta_{1}(x) \widehat{U}(x)}{1+b_{1}(x) \widehat{U}(x)} v_{3}(x, t)-\frac{\beta_{2}(x) \widehat{U}(x)}{1+b_{2}(x) \widehat{U}(x)} v_{2}(x, t) \\
\frac{\partial v_{2}}{\partial t}=\nabla & \cdot\left[D_{V}(x) \nabla v_{2}(x, t)\right] \\
& +\int_{\Omega} \Gamma(\tau, x, y) \frac{\beta_{1}(y) \widehat{U}(y)}{1+b_{1}(y) \widehat{U}(y)} v_{3}(t, y) d y \\
& +\int_{\Omega} \Gamma(\tau, x, y) \frac{\beta_{2}(y) \widehat{U}(y)}{1+b_{2}(y) \widehat{U}(y)} v_{2}(t, y) d y \\
& -d_{V}(x) v_{2}(x, t), \\
\frac{\partial v_{3}}{\partial t}= & \nabla \cdot\left[D_{\omega}(x) \nabla v_{3}(x, t)\right]+k(x) v_{2}(x, t)-d_{\omega}(x) v_{3}(x, t) .
\end{aligned}
$$

Substituting $v_{2}(x, t)=e^{\lambda t} \varphi_{1}(x)$ and $v_{3}(x, t)=e^{\lambda t} \varphi_{2}(x)$ into $v_{2}$ and $v_{3}$ of model (21) which is called the infectious compartments results in the following eigenvalue problem: 


$$
\begin{aligned}
\lambda \varphi_{1}(x)= & \nabla \cdot\left[D_{V}(x) \nabla \varphi_{1}(x)\right] \\
& +\int_{\Omega} \Gamma(\tau, x, y) \frac{\beta_{1}(y) \widehat{U}(y)}{1+b_{1}(y) \widehat{U}(y)} \varphi_{2}(y) d y \\
& +\int_{\Omega} \Gamma(\tau, x, y) \frac{\beta_{2}(y) \widehat{U}(y)}{1+b_{2}(y) \widehat{U}(y)} \varphi_{1}(y) d y \\
& -d_{V}(x) \varphi_{1}(x), \lambda \varphi_{2}(x)=\nabla \cdot\left[D_{\omega}(x) \nabla \varphi_{2}(x)\right] \\
& +k(x) \varphi_{1}(x)-d_{\omega}(x) \varphi_{2}(x), \\
{\left[D_{v}(x) \nabla \varphi_{1}(x)\right] \cdot v=} & {\left[D_{\omega}(x) \nabla \varphi_{2}(x)\right] \cdot v=0, } \\
& \forall x \in \partial \Omega, t>0, \varphi=\left(\varphi_{1}, \varphi_{2}\right) \in \mathbb{Z} \times \mathbb{Z} .
\end{aligned}
$$

By the similar argument to Theorem 7.6.1 in [39], it then follows that eigenvalue problem (22) has a principal eigenvalue $\lambda_{0}(\widehat{U}(x))$ with a positive eigenfunction.

We further consider the nonlocal eigenvalue problem associated with

$$
\begin{aligned}
& \lambda \varphi_{1}(x)= \nabla \cdot\left[D_{V}(x) \nabla \varphi_{1}(x)\right] \\
&+e^{-\lambda \tau} \int_{\Omega} \Gamma(\tau, x, y) \frac{\beta_{1}(y) \widehat{U}(y)}{1+b_{1}(y) \widehat{U}(y)} \varphi_{2}(y) d y \\
&+e^{-\lambda \tau} \int_{\Omega} \Gamma(\tau, x, y) \frac{\beta_{2}(y) \widehat{U}(y)}{1+b_{2}(y) \widehat{U}(y)} \varphi_{1}(y) d y \\
&-d_{V}(x) \varphi_{1}(x), \\
& \lambda \varphi_{2}(x)= \nabla \cdot\left[D_{\omega}(x) \nabla \varphi_{2}(x)\right]+k(x) \varphi_{1}(x) \\
&-d_{\omega}(x) \varphi_{2}(x), \\
& {\left[D_{v}(x) \nabla \varphi_{1}(x)\right] \cdot v=} {\left[D_{\omega}(x) \nabla \varphi_{2}(x)\right] \cdot v=0, } \\
& \quad \forall x \in \partial \Omega, t>0, \varphi=\left(\varphi_{1}, \varphi_{2}\right) \in \mathbb{Z} \times \mathbb{Z} .
\end{aligned}
$$

From Theorem 2.2 in [40], we get some useful information eigenvalue problem (23).

Lemma 2.1. The eigenvalue problem (23) has a principal eigenvalue $\bar{\lambda}_{0}(\widehat{U}(x))$ with a strictly positive eigenfunction, and for any $\tau \geq 0, \bar{\lambda}_{0}(\widehat{U}(\mathrm{x}))$ has the same sign as $\lambda_{0}(\widehat{U}(\mathrm{x}))$.

We adopt the same ideas as in [41-44] to define basic reproduction number of model (15). For this purpose, we define the positive linear operator as follows:

$\mathscr{H}(\varphi)(x)=\left(\mathscr{H}_{1}(\varphi)(x), \mathscr{H}_{2}(\varphi)(x)\right), \quad \forall \phi=\left(\varphi_{1}, \varphi_{2}\right) \in \mathbb{Z} \times \mathbb{Z}, x \in \bar{\Omega}$,

where

$$
\begin{aligned}
\mathscr{H}_{1}(\varphi)(x)= & \int_{\Omega} \Gamma(\tau, x, y) \frac{\beta_{1}(y) \hat{U}(y)}{1+b_{1}(y) \hat{U}(y)} \varphi_{2}(y) d y \\
& +\int_{\Omega} \Gamma(\tau, x, y) \frac{\beta_{2}(y) \hat{U}(y)}{1+b_{2}(y) \hat{U}(y)} \varphi_{1}(y) d y, \\
\mathscr{H}_{2}(\varphi)(x)= & k(x) \varphi_{1}(x) .
\end{aligned}
$$

In order to compute basic reproduction number $\mathscr{R}_{0}$, we assume that both infected cells and virus are near the infection-free steady state $E_{0}$ and introduce initial infected cells and virus at time $t=0$, where the distribution of initial infected cells and virus is described by $\left(\varphi_{1}(x), \varphi_{2}(x)\right)$. From model (15) and as time evolves, those distributions can reach

$$
[\mathscr{M}(t) \varphi](x)=\left(\left[\mathscr{M}_{2}(t) \varphi_{1}\right](x),\left[\mathscr{M}_{3}(t) \varphi_{2}\right](x)\right),
$$

at time $t$. Thus, the total distribution of new infected cells is

$$
\begin{aligned}
{\left[\int_{0}^{+\infty} \mathscr{H}_{1}(\mathscr{M} t)(\varphi) d t\right](x)=} & {\left[\int_{\tau}^{+\infty} \mathscr{H}_{1}(\mathscr{M}(t-\tau) \varphi) d t\right](x) } \\
= & \int_{0}^{+\infty} \int_{\Omega} \Gamma(\tau, x, y) \frac{\beta_{1}(y) \widehat{U}(y)}{1+b_{1}(y) \hat{U}(y)} \\
& \cdot\left[\mathscr{M}_{3}(t) \varphi_{2}\right](y) d y d \\
& +\int_{0}^{+\infty} \int_{\Omega} \Gamma(\tau, x, y) \frac{\beta_{2}(y) \hat{U}(y)}{1+b_{2}(y) \hat{U}(y)} \\
& \cdot\left[\mathscr{M}_{2}(t) \varphi_{1}\right](y) d y d t .
\end{aligned}
$$

Similarly, the total distribution of new virus can be described as

$$
\left[\int_{0}^{+\infty} \mathscr{H}_{2}(\mathscr{M}(t) \varphi) d t\right](x)=\int_{0}^{+\infty} k(x)\left[\mathscr{M}_{2}(t) \varphi_{1}\right](x) d t
$$

It then follows that

$$
\mathscr{L}(\varphi)=\int_{0}^{+\infty} \mathscr{H}(\mathscr{M}(t) \varphi) d t=\mathscr{H} \int_{0}^{+\infty}(\mathscr{M}(t) \varphi) d t
$$

where $\mathscr{L}$ is the next infection operator (see, for example, [41-44]). It maps the initial distribution of infected cells and virus to the total distribution of new infected cells and virus produced during the infection period.

By [41-44], we define the spectral radius of $\mathscr{L}$ as the basic reproduction number of model (15), namely,

$$
\mathscr{R}_{0}=r(\mathscr{L}) .
$$

In view of the general results developed in [42], we have the following observation.

Lemma 2.2. $\mathscr{R}_{0}-1$ has the same sign as $\lambda_{0}(\widehat{U}(x))$. 


\section{Threshold Dynamics in a Bounded Spatial Domain}

In this section, we study threshold dynamics in terms of $\mathscr{R}_{0}$ for model (15), assuming a bounded spatial domain $\Omega \subset \mathbb{R}^{n}$. We assume that all the parameters $\xi(x), \beta(x), a(x), b(x)$, $d_{U}(x), d_{V}(x), d_{\omega}(x)$, and $k(x)$ are spatially dependent. Assume these functions are positive, continuous, and bounded in the domain $\bar{\Omega}$. Let

$$
\begin{gathered}
\bar{\xi}=\max _{x \in \bar{\Omega}} \xi(x)>0, \\
\bar{\beta}=\max _{x \in \bar{\Omega}} \beta(x)>0, \\
\bar{k}=\max _{x \in \bar{\Omega}} k(x)>0, \\
\underline{a}=\min _{x \in \bar{\Omega}} a(x)>0, \\
\underline{b}=\min _{x \in \bar{\Omega}} b(x)>0, \\
\underline{d}_{U}=\min _{x \in \bar{\Omega}} d_{U}(x)>0, \\
\underline{d}_{V}=\min _{x \in \bar{\Omega}} d_{V}(x)>0, \\
\underline{d}_{\omega}=\min _{x \in \bar{\Omega}} d_{\omega}(x)>0 .
\end{gathered}
$$

For any $\phi=\left(\phi_{1}, \phi_{2}, \phi_{3}\right) \in \mathbb{C}_{+}$and $x \in \bar{\Omega}$, we define $\mathscr{F}=\left(\mathscr{F}_{1}, \mathscr{F}_{2}, \mathscr{F}_{3}\right): \mathbb{C}_{+} \rightarrow \mathbb{X}$ by

$$
\begin{aligned}
\mathscr{F}_{1}(\phi)(x)= & \xi(x)-\frac{\beta_{1}(x) \phi_{1}(0, x) \phi_{3}(0, x)}{1+a_{1}(x) \phi_{3}(0, x)+b_{1}(x) \phi_{1}(0, x)} \\
& -\frac{\beta_{2}(x) \phi_{1}(0, x) \phi_{2}(0, x)}{1+a_{2}(x) \phi_{2}(0, x)+b_{2}(x) \phi_{1}(0, x)}, \\
\mathscr{F}_{2}(\phi)(x)= & \int_{\Omega} \Gamma(\tau, x, y) \frac{\beta_{1}(y) \phi_{1}(-\tau, y) \phi_{3}(-\tau, y)}{1+a_{1}(x) \phi_{3}(-\tau, y)+b_{1}(x) \phi_{1}(-\tau, y)} d y \\
& +\int_{\Omega} \Gamma(\tau, x, y) \frac{\beta_{2}(y) \phi_{1}(-\tau, y) \phi_{2}(-\tau, y)}{1+a_{2}(x) \phi_{2}(-\tau, y)+b_{2}(x) \phi_{1}(-\tau, y)} d y, \\
\mathscr{F}_{3}(\phi)(x)= & k(x) \phi_{2}(0, x) .
\end{aligned}
$$

Obviously, $\mathscr{F}$ is locally Lipschitz. Then, model (15) can be rewritten as the following abstract functional differential equation:

$$
\begin{aligned}
\frac{d u}{d t} & =\mathscr{A} u+\mathscr{F}\left(u_{t}\right), \quad t>0, \\
u_{0} & =\phi \in \mathbb{C}_{+}
\end{aligned}
$$

where $u=(U, V, \omega)$.

Lemma 3.1. For any given $\phi \in \mathbb{C}_{+}$, there exists a unique mild solution $u(t, \cdot, \phi)$ of model (15) defined on its maximal interval of existence $\left[0, t_{\phi}\right)$ with $u_{0}=\phi$, where $t_{\phi} \leq \infty$. Further, $u(t, \cdot, \phi) \in \mathbb{C}_{+}, \forall t \in\left[0, t_{\phi}\right)$, and $u(t, \cdot, \phi)$ are a classical solution of model (15), $\forall t>\tau$.

Proof 1. For any $\phi=\left(\phi_{1}, \phi_{2}, \phi_{3}\right) \in \mathbb{C}_{+}$and for sufficiently small $k^{\prime} \geq 0$, we obtain that

$$
\begin{aligned}
\phi+k \mathscr{F}(\phi)(x)= & \left(\begin{array}{c}
\phi_{1}+k^{\prime} \xi(x)-\frac{k^{\prime} \beta_{1}(x) \phi_{1}(0, x) \phi_{3}(0, x)}{1+a_{1}(x) \phi_{3}(0, x)+b_{1}(x) \phi_{1}(0, x)}-\frac{k^{\prime} \beta_{2}(x) \phi_{1}(0, x) \phi_{2}(0, x)}{1+a_{2}(x) \phi_{2}(0, x)+b_{2}(x) \phi_{1}(0, x)} \\
\phi_{2}+\int_{\Omega} \frac{\Gamma(\tau, x, y) k^{\prime} \beta_{1}(y) \phi_{1}(-\tau, y) \phi_{3}(-\tau, y)}{1+a_{1}(y) \phi_{3}(-\tau, y)+b_{1}(y) \phi_{1}(-\tau, y)} d y+\int_{\Omega} \frac{\Gamma(\tau, x, y) k^{\prime} \beta_{2}(y) \phi_{1}(-\tau, y) \phi_{2}(-\tau, y)}{1+a_{2}(y) \phi_{2}(-\tau, y)+b_{2}(y) \phi_{1}(-\tau, y)} d y \\
\phi_{3}+k^{\prime} k(x) \phi_{2}(0, x)
\end{array}\right) \geq\left(\begin{array}{c}
0 \\
0 \\
0
\end{array}\right) .
\end{aligned}
$$

Thus, $\phi+k^{\prime} \mathscr{F}(\phi)(x) \in \mathbb{C}_{+}$. This implies that $\lim _{k^{\prime} \rightarrow 0^{+}}\left(1 / k^{\prime}\right)$ $\operatorname{dist}\left(\phi+k^{\prime} \mathscr{F}(\phi)(x), \mathbb{C}_{+}\right)=0$, for all $\phi \in \mathbb{C}_{+}$. From Corollary 4 in [45] (see, also [46], Corollary 8.1.3), we obtain the conclusion stated in Lemma 3.1. The proof is completed.

We are now in the position to study the well-posedness of model (15) in the sense of the following theorem.
Theorem 3.1. For any $\phi=\left(\phi_{1}, \phi_{2}, \phi_{3}\right) \in \mathbb{C}_{+}$, model (15) has a unique solution $u(t, \cdot, \phi)$ on $[0, \infty)$ with $u_{0}=\phi$. Moreover, the solution semiflow $\Phi(t)=u(t, \cdot, \phi): \mathbb{C}_{+} \rightarrow \mathbb{C}_{+}$has a compact global attractor for $t \geq 0$.

Proof 2. It is easy to see that model (15) defines a semiflow $\Phi(t):=u_{t}(\cdot): \mathbb{C}_{+} \rightarrow \mathbb{C}_{+}$by 


$$
(\Phi(t) \phi)(\theta, x)=u(t+\theta, x, \phi), \quad \forall \theta \in[-\tau, 0], x \in \bar{\Omega}, t>0 .
$$

For any fixed $\phi=\left(\phi_{1}, \phi_{2}, \phi_{3}\right) \in \mathbb{C}_{+} \rightarrow \mathbb{C}_{+}$, from the first equation of model (15), we obtain that

$$
\frac{\partial U(x, t)}{\partial t} \leq \nabla \cdot\left[D_{U}(x) \nabla U(x, t)\right]+\bar{\xi}-\underline{d}_{U} U(x, t) .
$$

From the comparison principle, we easily get that there exists $t_{1}(\phi)>0$ such that $U(x, t) \leq\left(2 \bar{\xi} / \underline{d}_{U}\right)=: \mathscr{B}_{1}$ for $t>t_{1}$. From the second equation of model (15), we have that

$\frac{\partial V(x, t)}{\partial t} \leq \nabla \cdot\left[D_{V}(x) \nabla V(x, t)\right]+\frac{\bar{\beta}_{1} \mathscr{B}_{1}}{\underline{a}_{1}}+\frac{\bar{\beta}_{2} \mathscr{B}_{1}}{\underline{a}_{2}}-\underline{d}_{V} V(x, t)$.

There exists $t_{2}(\phi)>0$ such that $V(x, t) \leq\left(2 \bar{\beta}_{1} \mathscr{B}_{1} / \underline{a}_{1} \underline{d}_{V}\right)+$ $\left(2 \bar{\beta}_{2} \mathscr{B}_{1} / \underline{a}_{2} \underline{d}_{V}\right)=: \mathscr{B}_{2}$ for $t>t_{2}$. From the third equation of model (15), we get

$$
\frac{\partial \omega(x, t)}{\partial t} \leq \nabla \cdot\left[D_{\omega}(x) \nabla \omega(x, t)\right]+\bar{k} \mathscr{B}_{2}-\underline{d}_{\omega} \omega(x, t) .
$$

There also exists $t_{3}(\phi)>0$ such that $\omega(x, t) \leq\left(2 \bar{k}_{\mathscr{B}} /\right.$ $\left.\underline{d}_{\omega}\right)=: \mathscr{B}_{3}$ for $t>t_{3}$.

Consequently, the existence of solutions $u(t, \cdot, \phi)$ of model (15) claimed in Lemma 3.1 is indeed global (i.e., $\left.t_{\phi}=\infty\right)$. The solution semiflow $\Phi(t): \mathbb{C}_{+} \rightarrow \mathbb{C}_{+}$is point dissipative. Moreover, by Theorem 2.2.6 in [46], we have that $\Phi(t): \mathbb{C}_{+} \rightarrow \mathbb{C}_{+}$is compact for any $t>\tau$. Thus, from Theorem 3.4.8 in [47], we know that $\Phi(t): \mathbb{C}_{+} \rightarrow \mathbb{C}_{+}$has a compact global attractor in $\mathbb{C}_{+}$for $t \geq 0$. We complete the proof.

The following results will play an important role in establishing uniform persistence of model (15).

Lemma 3.2. Let $u(t, \cdot, \phi)$ be the solution of model (15) with $u(0, \cdot, \phi)=\phi \in \mathbb{C}_{+}$, then we have the following:

(i) If there exists some $t_{0}>0$ such that $V\left(t_{0}, \cdot, \phi\right) \neq 0$ and $\omega\left(t_{0}, \cdot, \phi\right) \equiv 0$, then

$$
\begin{aligned}
& V(t, \cdot, \phi)>0, \\
& \omega(t, \cdot, \phi)>0,
\end{aligned}
$$

for all $t>t_{0}, x \in \bar{\Omega}$.

(ii) It holds that $U(t, \cdot, \phi)>0$ for any $t>0, x \in \bar{\Omega}$, and

$$
\liminf _{t \rightarrow \infty} U(t, \cdot, \phi) \geq \frac{\underline{\xi}}{d_{U}+\left(\bar{\beta}_{1} / \underline{a}_{1}\right)+\left(\bar{\beta}_{2} / \underline{a}_{2}\right)},
$$

uniformly for $x \in \bar{\Omega}$.

Proof 3. From the second and third equations of model (15), it follows that

$$
\begin{aligned}
& \frac{\partial V(x, t)}{\partial t} \geq \nabla \cdot\left[D_{V}(x) \nabla V(x, t)\right]-d_{V}(x) V(x, t), \\
& \frac{\partial \omega(x, t)}{\partial t} \geq \nabla \cdot\left[D_{\omega}(x) \nabla \omega(x, t)\right]-d_{\omega}(x) \omega(x, t),
\end{aligned}
$$

with the following boundary conditions

$$
\frac{\partial V(x, t)}{\partial v}=\frac{\partial \omega(x, t)}{\partial v}=0, \quad x \in \partial \Omega .
$$

If $V\left(t_{0}, \cdot, \phi\right) \equiv 0$ and $\omega\left(t_{0}, \cdot, \phi\right) \neq 0$ for some $t_{0} \geq 0$, it follows from the strong maximum principle (see, for example, [48] p. 172, Theorem 4) and the Hopf boundary lemma (see, for example, [48] p. 170, Theorem 3) that $V(t, \cdot, \phi)>0$, $M(t, \cdot, \phi)>0$, and $\omega(t, \cdot, \phi)>0$ for $t>t_{0}, x \in \bar{\Omega}$, that is, the conclusion $(i)$ holds.

From the first equation of model (15), we get

$$
\frac{\partial U(x, t)}{\partial t} \geq D_{0} \Delta U+\underline{\xi}-\left(d_{U}+\frac{\bar{\beta}_{1}}{\underline{a}_{1}}+\frac{\bar{\beta}_{2}}{\underline{a}_{2}}\right) U(x, t) .
$$

Let $v(x, t, \phi)$ be the solution of

$$
\begin{aligned}
\frac{\partial v(x, t)}{\partial t} & =D_{0} \Delta v+\underline{\xi}-\left(d_{U}+\frac{\bar{\beta}_{1}}{\underline{a}_{1}}+\frac{\bar{\beta}_{2}}{\underline{a}_{2}}\right) v(x, t), \\
\frac{\partial v}{\partial v} & =0, \quad x \in \partial \Omega, \\
v(0, x) & =\phi(0, x) .
\end{aligned}
$$

It then follows that $U(t, x, \phi) \geq v(t, x, \phi)>0$ for all $t>0$ and $x \in \bar{\Omega}$. It follows from the standard parabolic comparison principle that $\liminf _{t \rightarrow \infty} U(t, \cdot, \phi) \geq \underline{\xi} /\left(d_{U}+\left(\bar{\beta}_{1} / \underline{a}_{1}\right)+\left(\bar{\beta}_{2} / \underline{a}_{2}\right)\right)$ uniformly for $x \in \bar{\Omega}$. The proof is completed.

The following theorem indicates that $\mathscr{R}_{0}$ is a threshold quantity for virus extinction or persistence.

Theorem 3.2. Suppose $u(x, t, \phi)$ is the solution of model (15) with $u_{0}=\phi \in \mathbb{C}_{+}$.. Then, the following statements hold:

(i) If $\mathscr{R}_{0}<1$, then the infection-free steady state $E_{0}$ of model (15) is globally attractive.

(ii) If $\mathscr{R}_{0}>1$, then model (15) has at least one coexistence steady state and there exists $\varsigma>0$ such that any nonnegative solution $u(t, x, \phi)$ with $\phi_{2}(0) \neq 0$ and $\phi_{3}(0) \neq 0$, we get

$$
\begin{aligned}
& \liminf _{t \rightarrow \infty} V(x, t) \geq \varsigma, \\
& \liminf _{t \rightarrow \infty} \omega(x, t) \geq \varsigma,
\end{aligned}
$$

uniformly for all $x \in \bar{\Omega}$.

Proof 4. From Lemma 2.2, we easily obtain that $\lambda_{0}\left(\widehat{U}_{0}\right)<0$ when $\mathscr{R}_{0}<1$. Since $\lim _{\varepsilon \rightarrow 0} \lambda_{0}\left(\widehat{U}_{0}+\varepsilon\right)=\lambda_{0}\left(\widehat{U}_{0}\right)<0$, there exists $\varepsilon_{0}>0$ sufficiently small such that $\lambda_{0}\left(\widehat{U}_{0}+\varepsilon_{0}\right)<0$. For fixed 
$\varepsilon_{0}>0$, there exists $T_{2}>0$ such that $U(x, t) \leq \widehat{U}_{0}+\varepsilon_{0}$ for all $t \geq T_{2}, x \in \bar{\Omega}$. Therefore, for all $t \geq T_{2}$, from the second and third equations of model (15), it follows that

$$
\begin{aligned}
\frac{\partial V}{\partial t} \leq & \nabla \cdot\left[D_{V}(x) \nabla V(x, t)\right] \\
& +\int_{\Omega} \Gamma(\tau, x, y) \frac{\beta_{1}(y)\left(\widehat{U}+\varepsilon_{0}\right)}{1+b_{1}(y)\left(\widehat{U}+\varepsilon_{0}\right)} \omega(y, t-\tau) d y \\
& +\int_{\Omega} \Gamma(\tau, x, y) \frac{\beta_{2}(y)\left(\widehat{U}+\varepsilon_{0}\right)}{1+b_{2}(y)\left(\widehat{U}+\varepsilon_{0}\right)} V(y, t-\tau) d y \\
& -d_{V}(x) V(x, t), \\
\frac{\partial \omega}{\partial t} \leq & \nabla \cdot\left[D_{\omega}(x) \nabla \omega(x, t)\right]+k(x) V(x, t)-d_{\omega}(x) \omega(x, t) .
\end{aligned}
$$

According to Lemma 2.1, we get $\bar{\lambda}_{0}\left(\widehat{U}+\varepsilon_{0}\right)<0$, and there exists a positive eigenfunction $\psi_{0}$ corresponding to $\bar{\lambda}_{0}$ $\left(\widehat{U}+\varepsilon_{0}\right)<0$. It then obtains the following linear system:

$$
\begin{aligned}
\frac{\partial u_{2}}{\partial t}= & \nabla \cdot\left[D_{V}(x) \nabla u_{2}(x, t)\right] \\
& +\int_{\Omega} \Gamma(\tau, x, y) \frac{\beta_{1}(y)\left(\widehat{U}+\varepsilon_{0}\right)}{1+b_{1}(y)\left(\widehat{U}+\varepsilon_{0}\right)} u_{3}(y, t-\tau) d y \\
& +\int_{\Omega} \Gamma(\tau, x, y) \frac{\beta_{2}(y)\left(\widehat{U}+\varepsilon_{0}\right)}{1+b_{2}(y)\left(\widehat{U}+\varepsilon_{0}\right)} u_{2}(y, t-\tau) d y-d_{V}(x) u_{2}, \\
\frac{\partial u_{3}}{\partial t}= & \nabla \cdot\left[D_{\omega}(x) \nabla u_{3}(x, t)\right]+k(x) u_{2}-d_{\omega}(x) u_{3},
\end{aligned}
$$

with the following boundary conditions

$$
\frac{\partial u_{2}}{\partial v}=\frac{\partial u_{3}}{\partial v}=0, \quad x \in \bar{\Omega},
$$

admitting a solution $u(t, x)=e^{\bar{\lambda}_{0}\left(\widehat{U}_{0}+\varepsilon_{0}\right) t} \psi_{0}(x)$. Then, for any given $\phi \in \mathbb{C}_{+}$, there exists $\alpha^{\prime}>0$ such that

$$
(V(t, \cdot, \phi), \omega(t, \cdot, \phi)) \leq \alpha^{\prime} u(t, \cdot), \quad t \in\left[T_{2}-\tau, T_{2}\right] .
$$

By the comparison principle, it follows that

$$
(V(t, x, \phi), \omega(t, x, \phi)) \leq \alpha^{\prime} e^{\lambda_{0}\left(\hat{U}_{0}+\varepsilon_{0}\right) t} \psi_{0}(x), \quad \forall t \geq T_{2} .
$$

Thus, $\lim _{t \rightarrow+\infty}(V(t, x, \phi), \omega(t, x, \phi))=0$ uniformly for $x \in \bar{\Omega}$. Hence, by [35], it then follows that $\lim _{t \rightarrow+\infty} U(t, x, \phi)=\widehat{U}_{0}(x)$ uniformly for $\forall x \in \bar{\Omega}$, which ends the proof of Part (i).

For $\mathscr{R}_{0}>1$, we employ the persistence theory in [49] to study uniform persistence of model (15). Define

$$
\mathbb{X}_{0}=\left\{\phi=\left(\phi_{1}, \phi_{2}, \phi_{3}\right) \in \mathbb{C}_{+}: \phi_{2}(0) \neq 0 \text { and } \phi_{3}(0) \equiv 0\right\} .
$$

Obviously, we have that

$$
\partial \mathbb{X}_{0}:=\mathbb{C}_{+} \backslash \mathbb{X}_{0}=\left\{\phi \in \mathbb{C}_{+}: \phi_{2}(0) \equiv 0 \text { or } \phi_{3}(0) \equiv 0\right\} .
$$

In view of Lemma 3.2, it then follows that $\mathbb{X}_{0}$ is positively invariant for the solution semiflow $\Phi(t)$. Define

$$
\mathscr{W}_{\partial}:=\left\{\phi \in \partial \mathbb{X}_{0}: \Phi(t) \phi \in \partial \mathbb{X}_{0}, \quad \forall t \geq 0\right\}
$$

Let $\omega(\phi)$ be the omega limit set of the orbit of $\Phi(t)$ through $\phi \in \mathbb{C}_{+}$, and set $\mathscr{W}_{1}:=\{(\widehat{U}(x), 0,0)\}$.

Claim 1. $\cup_{\phi \in \mathscr{W}_{\partial}} \omega(\phi)=\mathscr{W}_{1}$.

For any given $\phi \in \mathscr{W}_{\partial}$, it follows that $u_{t}(\phi) \in \partial \mathbb{X}_{0}$ for all $t \geq 0$. Since $u_{t}(\phi)=u(t, \cdot, \phi)$, we easily have that for each $t \geq 0$ either $V(t, \cdot, \phi) \equiv 0$ or $\omega(t, \cdot, \phi) \equiv 0$. For the case where $V(t, \cdot, \phi) \equiv 0, \forall t \geq 0$, combined with the third equation of model (15), it can be obtained that $\lim _{t \rightarrow+\infty} \omega(t, \cdot, \phi)=0$ uniformly for $x \in \bar{\Omega}$. Further, from the first equation of model (15) and the theory of asymptotically autonomous semiflows [50], it then follows that $\lim _{t \rightarrow+\infty} U(t, \cdot, \phi)=\widehat{U}(x)$ uniformly for $x \in \bar{\Omega}$. In the case where $V\left(t_{0}, \cdot, \phi\right) \equiv 0$ for some $t_{0}>0$, by parabolic maximum principle, we have that $V(t, \cdot, \phi)>0$ for all $t>t_{0}$ and $x \in \bar{\Omega}$. Therefore, we get $\omega(t, \cdot, \phi) \equiv 0$ for all $t \geq$ $t_{0}$. From the third equation of model (15), we obtain that $V(t, \cdot, \phi) \equiv 0$ for all $t \geq t_{0}$, which is a contradiction. Hence, we get $\cup_{\phi \in \mathscr{W}_{2}} \omega(\phi)=\mathscr{W}_{1}$. We complete the proof of Claim 1 .

Claim 2. Similar to the proof of Lemma 3.3 in [17], we easily get that $E_{0}$ is a uniform weak repeller in the sense that

$$
\limsup _{t \rightarrow \infty}\|u(t, \cdot, \phi)-(\widehat{U}(x), 0,0)\|_{\mathbb{C}_{+}} \geq \chi .
$$

We define a continuous function $p: \mathbb{C}_{+} \rightarrow \mathbb{R}^{+}$by

$$
p(\phi)=\min \left\{\min _{x \in \bar{\Omega}} \phi_{2}(0)(x), \min _{x \in \bar{\Omega}} \phi_{3}(0)(x)\right\}, \quad \forall \phi \in \mathbb{C}_{+} .
$$

We easily verify that $p^{-1}(0,+\infty) \subset \mathbb{X}_{0}$. By Lemma 3.3, $p$ has the property that if either $p(\phi)=0$ and $\phi \in \mathbb{X}_{0}$ or $p(\phi)>0$, then $p(\Phi(t)(\phi))>0$ for all $t>0$. Consequently, we have that $p$ is a generalized distance function for the semiflow $\Phi(t): \mathbb{C}_{+} \rightarrow \mathbb{C}_{+}$(see [49]). From the above claims, it follows that any forward orbit of $\Phi(t)$ in $\mathscr{W}_{\partial}$ converges to $\mathscr{W}_{1}$ which is isolated in $\mathbb{C}_{+}$, and $W^{s}\left(\mathscr{W}_{1}\right)$ $\cap \mathbb{X}_{0}=0$, where $W^{s}\left(\mathscr{W}_{1}\right)$ is the stable set of $\mathscr{W}_{1}$ (see, for example, [49]). Further, we find that no subset of $\mathscr{W}_{1}$ forms a cycle in $\partial \mathbb{X}_{0}$ from $E_{0}$ to $E_{0}$. By Theorem 3 in [49], we prove that there exists $\varsigma>0$ such that $\min \{p$ $(\psi): \psi \in \omega(\phi)\}>\varsigma$ for any $\phi \in \mathbb{X}_{0}$. In view of Theorem 1.3.6 in [51], we obtain that model (15) has at least one coexistence steady state if $\mathscr{R}_{0}>1$. The proof is completed.

\section{Discussions and Biological Implications}

This paper is devoted to develop a nonlocal and time-delayed reaction-diffusion model for HIV infection within host cell-to-cell viral transmissions. For model (15), the virus 
dynamics are fully determined by an important parameter which is called basic reproduction number $\mathscr{R}_{0}$, in the following sense: if $\mathscr{R}_{0}<1$, the infection-free steady state $E_{0}$ is globally attractive, implying infection becomes extinct; while if $\mathscr{R}_{0}>1$, the virus will persist in the host environment.

\section{Conflicts of Interest}

The authors declare that there are no conflicts of interest regarding the publication of this paper.

\section{Acknowledgments}

This work is supported by Shandong Provincial Natural Science Foundation (no. ZR2015AQ001), the National Natural Science Foundation of China (no. 11371230), the SDUST Research Fund (2014TDJH102), Joint Innovative Center for Safe and Effective Mining Technology and Equipment of Coal Resources by Shandong Province, and SDUST.

\section{References}

[1] R. Anderson and R. May, Infectious Diseases of Humans: Dynamics and Control, Oxford University Press, Oxford, 1991.

[2] X. Meng, S. Zhao, T. Feng, and T. Zhang, "Dynamics of a novel nonlinear stochastic SIS epidemic model with double epidemic hypothesis," Journal of Mathematical Analysis and Applications, vol. 433, no. 1, pp. 227-242, 2016.

[3] A. Miao, X. Wang, T. Zhang, W. Wang, and B. G. Sampath Aruna Pradeep, "Dynamical analysis of a stochastic SIS epidemic model with nonlinear incidence rate and double epidemic hypothesis," Advances in Difference Equations, vol. 2017, no. 1, 226 pages, 2017.

[4] Z. Ma, Y. Zhou, and J. Wu, Modeling and Dynamics of Infectious Diseases, World Scientific Press, Singapor, 2009.

[5] T. Zhang, X. Meng, T. Zhang, and Y. Song, "Global dynamics for a new high-dimensional SIR model with distributed delay," Applied Mathematics and Computation, vol. 218, no. 24, pp. 11806-11819, 2012.

[6] A. Miao, J. Zhang, T. Zhang, and B. G. S. A. Pradeep, “Threshold dynamics of a stochastic SIR model with vertical transmission and vaccination," Computational and Mathematical Methods in Medicine, vol. 2017, Article ID 4820183, 10 pages, 2017.

[7] X. Leng, T. Feng, and X. Meng, "Stochastic inequalities and applications to dynamics analysis of a novel SIVS epidemic model with jumps," Journal of Inequalities and Applications, vol. 2017, no. 1, 138 pages, 2017.

[8] W. Zhao, T. Zhang, Z. Chang, X. Meng, and Y. Liu, "Dynamical analysis of SIR epidemic models with distributed delay," Journal of Applied Mathematics, vol. 2013, Article ID 154387, 15 pages, 2013.

[9] F. Li, X. Meng, and X. Wang, "Analysis and numerical simulations of a stochastic SEIQR epidemic system with quarantineadjusted incidence and imperfect vaccination," Computational and Mathematical Methods in Medicine, vol. 2018, Article ID 7873902, 14 pages, 2018.

[10] H. Qi, L. Liu, and X. Meng, "Dynamics of a non-autonomous stochastic SIS epidemic model with double epidemic hypothesis," Complexity, vol. 2017, Article ID 4861391, 14 pages, 2017.
[11] S. Zhang, X. Meng, and X. Wang, "Application of stochastic inequalities to global analysis of a nonlinear stochastic SIRS epidemic model with saturated treatment function," Advances in Difference Equations, vol. 2018, no. 1, 50 pages, 2018.

[12] S. Guo and W. Ma, "Global behavior of delay differential equations model of HIV infection with apoptosis," Discrete and Continuous Dynamical Systems-Series B, vol. 21, no. 1, pp. 103-119, 2016.

[13] R. Xu, "Global dynamics of a delayed HIV-1 infection model with absorption and saturation infection," International Journal of Biomathematics, vol. 5, no. 3, article 1260012, 2012.

[14] G. Huang, X. Liu, and Y. Takeuchi, "Lyapunov functions and global stability for age-structured HIV infection model," SIAM Journal on Applied Mathematics, vol. 72, no. 1, pp. 25-38, 2012.

[15] X. Meng, Y. Yang, and S. Zhao, "Adaptive evolution of virulence-related traits in a susceptible-infected model with treatment," Abstract and Applied Analysis, vol. 2014, Article ID 891401, 10 pages, 2014.

[16] T. Zhang, X. Liu, X. Meng, and T. Zhang, "Spatio-temporal dynamics near the steady state of a planktonic system," Computers \& Mathematics with Applications, vol. 75, no. 12, pp. 4490-4504, 2018.

[17] W. Wang and T. Zhang, "Caspase-1-mediated pyroptosis of the predominance for driving $\mathrm{CD} 4^{+} \mathrm{T}$ cells death: a nonlocal spatial mathematical model," Bulletin of Mathematical Biology, vol. 80, no. 3, pp. 540-582, 2018.

[18] K. Wang and W. Wang, "Propagation of HBV with spatial dependence," Mathematical Biosciences, vol. 210, no. 1, pp. 78-95, 2007.

[19] C. M. Brauner, D. Jolly, L. Lorenzi, and R. Thiebaut, "Heterogeneous viral environment in a HIV spatial model," Discrete and Continuous Dynamical Systems-Series B, vol. 15, no. 3, pp. 545-572, 2011.

[20] F.-B. Wang, Y. Huang, and X. Zou, "Global dynamics of a PDE in-host viral model," Applicable Analysis, vol. 93, no. 11, pp. 2312-2329, 2014.

[21] K. Hattaf and N. Yousfi, "Global dynamics of a delay reactiondiffusion model for viral infection with specific functional response," Computational and Applied Mathematics, vol. 34, no. 3, pp. 807-818, 2015.

[22] W. Wang and W. Ma, "A diffusive HIV infection model with nonlocal delayed transmission," Applied Mathematics Letters, vol. 75, pp. 96-101, 2018.

[23] W. Wang and W. Ma, "Travelling wave solutions for a nonlocal dispersal HIV infection dynamical model," Journal of Mathematical Analysis and Applications, vol. 457, no. 1, pp. 868-889, 2018.

[24] W. Wang and W. Ma, "Hepatitis C virus infection is blocked by hmgbl: a new nonlocal and time-delayed reaction-diffusion model," Applied Mathematics and Computation, vol. 320, pp. 633-653, 2018.

[25] X. Lai and X. Zou, "Repulsion effect on superinfecting virions by infected cells," Bulletin of Mathematical Biology, vol. 76, no. 11, pp. 2806-2833, 2014.

[26] Z. Wang and R. Xu, "Stability and traveling waves of an epidemic model with relapse and spatial diffusion," Journal of Applied Analysis \& Computation, vol. 4, no. 3, pp. 307-322, 2014.

[27] W. Wang, W. Ma, and X. Lai, "Repulsion effect on superinfecting virions by infected cells for virus infection dynamic model 
with absorption effect and chemotaxis," Nonlinear Analysis: Real World Applications, vol. 33, pp. 253-283, 2017.

[28] T. Zhang, X. Meng, and T. Zhang, "Global dynamics of a virus dynamical model with cell-to-cell transmission and cure rate," Computational and Mathematical Methods in Medicine, vol. 2015, Article ID 758362, 8 pages, 2015.

[29] T. Zhang, X. Meng, and T. Zhang, "Global analysis for a delayed SIV model with direct and environmental transmissions," Journal of Applied Analysis \& Computation, vol. 6, no. 2, pp. 479-491, 2016.

[30] M. Nowak and R. May, Virus Dynamics, Oxford University Press, Oxford, 2000.

[31] W. Hübner, G. P. McNerney, P. Chen et al., "Quantitative 3D video microscopy of HIV transfer across T cell virological synapses," Science, vol. 323, no. 5922, pp. 1743-1747, 2009.

[32] J. Wang, J. Yang, and T. Kuniya, "Dynamics of a PDE viral infection model incorporating cell-to-cell transmission," Journal of Mathematical Analysis and Applications, vol. 444, no. 2, pp. 1542-1564, 2016.

[33] N. F. Britton, "Spatial structures and periodic travelling waves in an integro-differential reaction-diffusion population model," SIAM Journal on Applied Mathematics, vol. 50, no. 6, pp. 1663-1688, 1990.

[34] S. Gourley and J. Wu, "Delayed non-local diffusive systems in biological invasion and disease spread," Nonlinear Dynamics and Evolution Equations, vol. 48, 2006.

[35] Z. Guo, F.-B. Wang, and X. Zou, "Threshold dynamics of an infective disease model with a fixed latent period and nonlocal infections," Journal of Mathematical Biology, vol. 65, no. 6-7, pp. 1387-1410, 2012.

[36] J. R. Beddington, "Mutual interference between parasites or predators and its effect on searching efficiency," The Journal of Animal Ecology, vol. 44, no. 1, p. 331, 1975.

[37] D. L. DeAngelis, R. A. Goldstein, and R. V. O'Neill, “A model for tropic interaction," Ecology, vol. 56, no. 4, pp. 881-892, 1975.

[38] A. Friedman, Partial Differential Equations of Parabolic Type, Prentice-Hall, Englewood Cliffs, 1964.

[39] H. Smith, Monotone Dynamical Systems: an Introduction to the Theory of Competitive and Cooperative Systems, no. 41, 1995American Mathematical Society, Providence, RI, 1995.

[40] H. R. Thieme and X.-Q. Zhao, "A non-local delayed and diffusive predator-prey model," Nonlinear Analysis: Real World Applications, vol. 2, no. 2, pp. 145-160, 2001.

[41] O. Diekmann, J. A. P. Heesterbeek, and J. A. J. Metz, "On the definition and the computation of the basic reproduction ratio R0 in models for infectious diseases in heterogeneous populations," Journal of Mathematical Biology, vol. 28, no. 4, 1990.

[42] H. R. Thieme, "Spectral bound and reproduction number for infinite-dimensional population structure and time heterogeneity," SIAM Journal on Applied Mathematics, vol. 70, no. 1, pp. 188-211, 2009.

[43] P. van den Driessche and J. Watmough, "Reproduction numbers and sub-threshold endemic equilibria for compartmental models of disease transmission," Mathematical Biosciences, vol. 180, no. 1-2, pp. 29-48, 2002.

[44] W. Wang and X.-Q. Zhao, "A nonlocal and time-delayed reaction-diffusion model of dengue transmission," SIAM Journal on Applied Mathematics, vol. 71, no. 1, pp. 147-168, 2011.
[45] R. H. Martin and H. L. Smith, "Abstract functional-differential equations and reaction-diffusion systems," Transactions of the American Mathematical Society, vol. 321, no. 1, pp. 1-44, 1990.

[46] J. Wu, Theory and Applications of Partial Functional Differential Equations, Springer New York, New York, 1996.

[47] J. K. Hale, "Asymptotic behavior of dissipative systems," American Mathematical Society Providence Ri, vol. 25, p. 198, 1987.

[48] M. Protter and H. Weinberger, Maximum Principles in Differential Equations, Springer New York, New York, 1984.

[49] H. L. Smith and X.-Q. Zhao, "Robust persistence for semidynamical systems," Nonlinear Analysis: Theory, Methods of Applications, vol. 47, no. 9, pp. 6169-6179, 2001.

[50] H. R. Thieme, "Convergence results and a Poincaré-Bendixson trichotomy for asymptotically autonomous differential equations," Journal of Mathematical Biology, vol. 30, no. 7, 1992.

[51] X. Q. Zhao, Dynamical Systems in Population Biology, Springer New York, New York, 2003. 


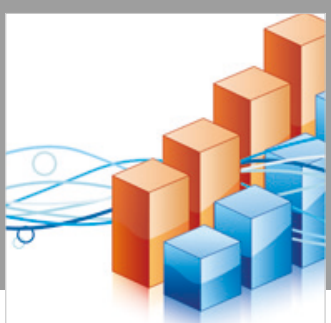

Advances in

Operations Research

\section{-n-m}
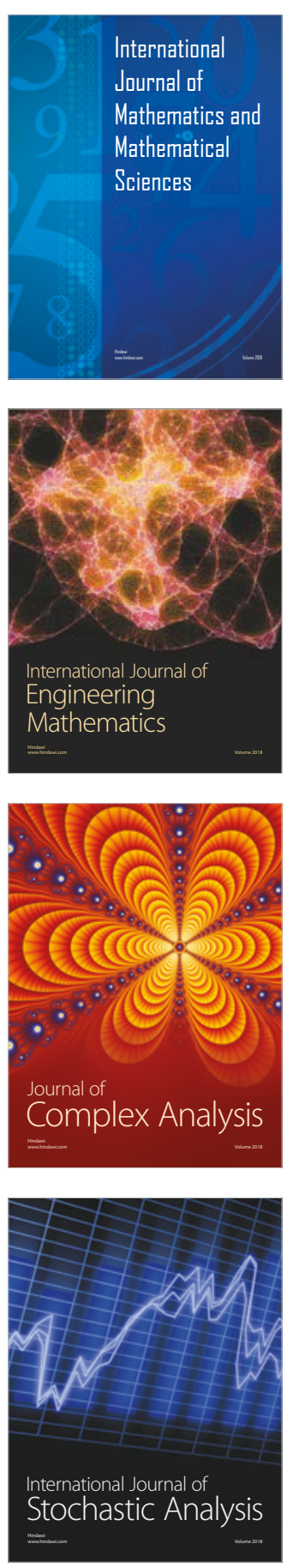
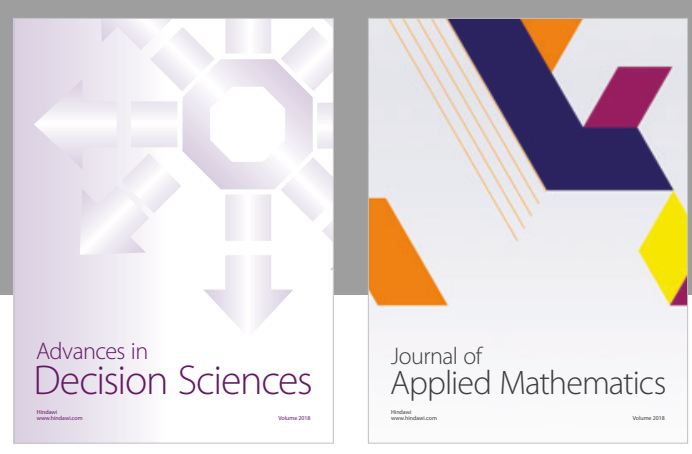

Journal of

Applied Mathematics
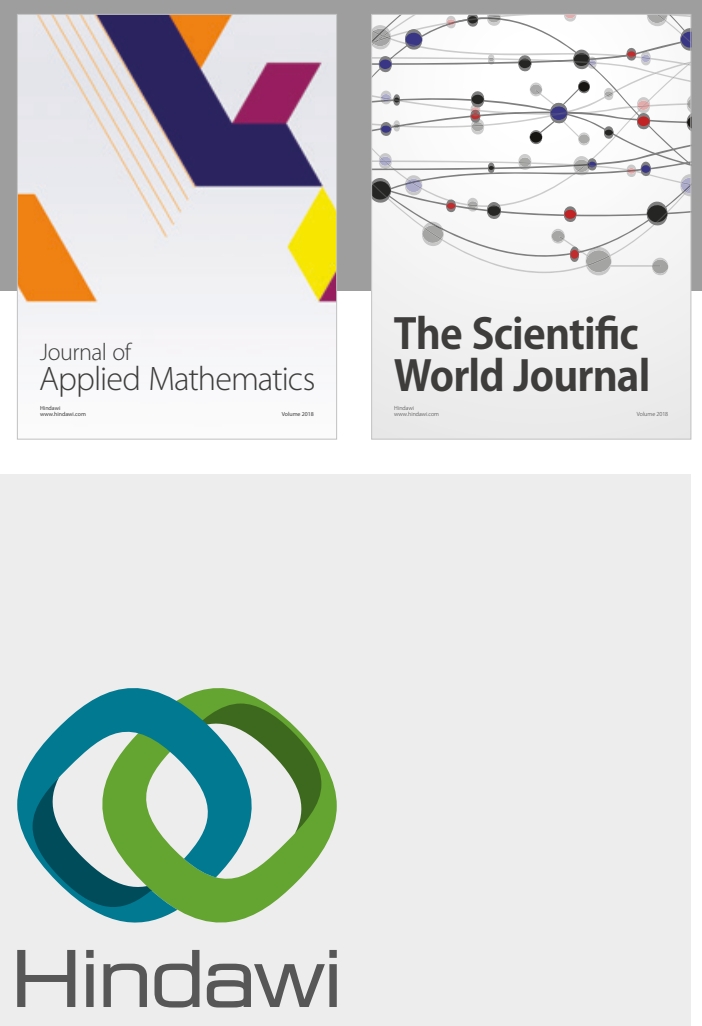

Submit your manuscripts at

www.hindawi.com

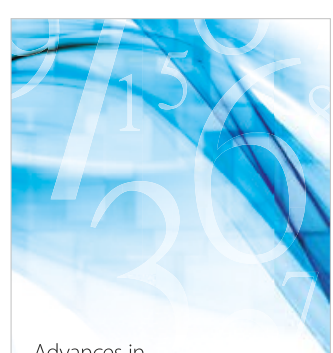

Advances in
Numerical Analysis
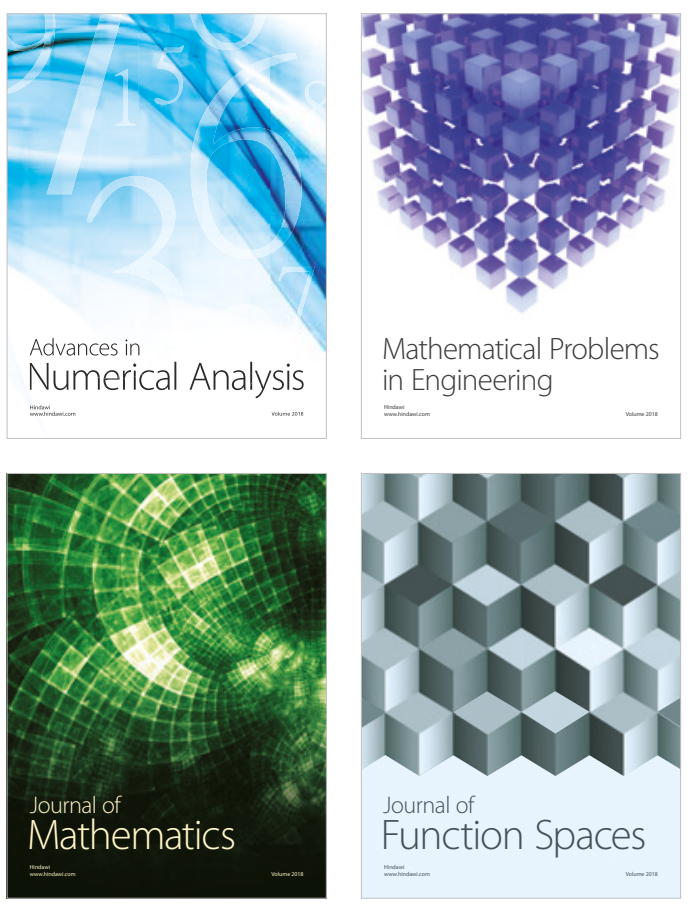

Mathematical Problems in Engineering

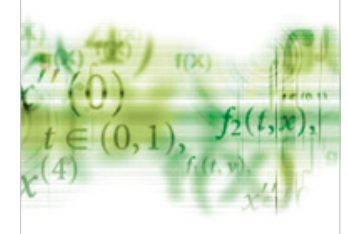

International Journal of

Differential Equations

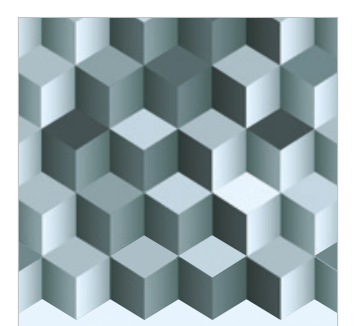

Journal of

Function Spaces
The Scientific

World Journal

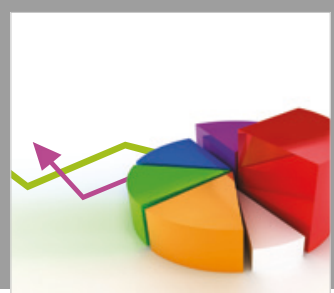

Journal of

Probability and Statistics
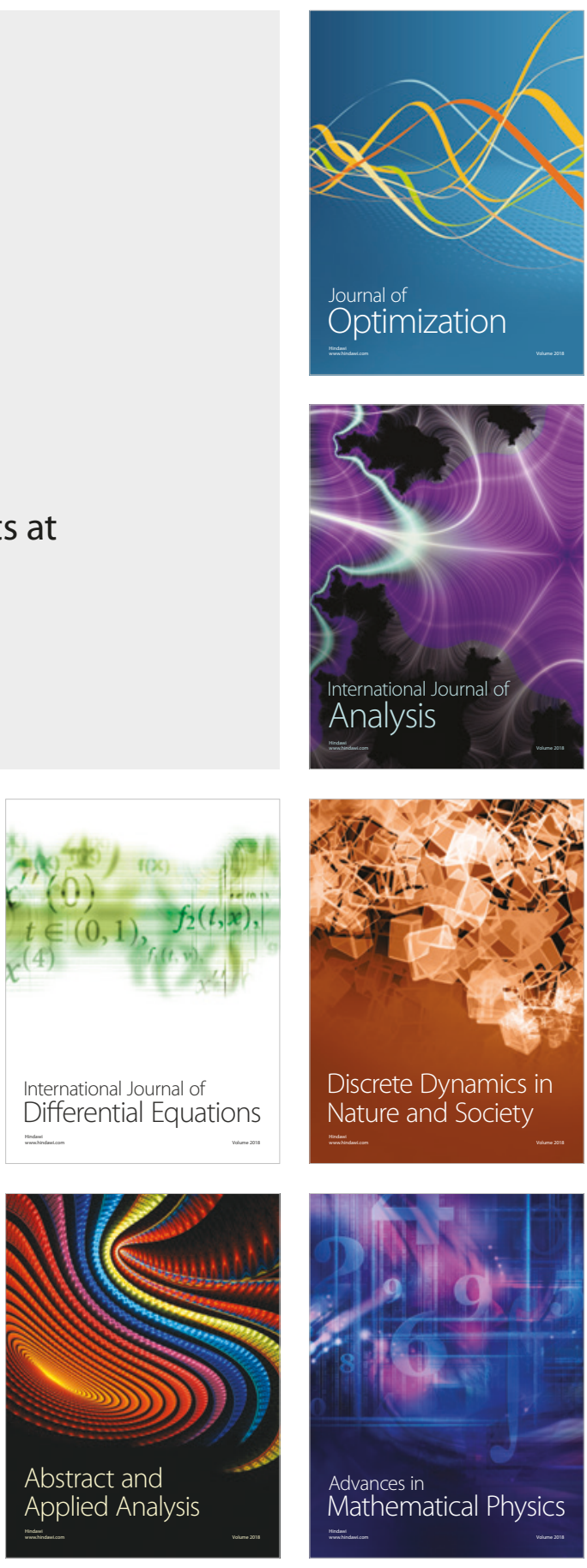